\title{
Design and Implementation of the Intelligent Music Recommender System on the Mobile Phone
}

\author{
Ki-Young Lee ${ }^{1}$, Jeong-Jin Kang ${ }^{2}$, Aleum Kim ${ }^{1}$, Myung-Jae Lim ${ }^{1, *}$, Yong-Soon $\mathrm{Im}^{3}$, \\ Sung-Jai Choi ${ }^{4}$ and Jong-Jin Park ${ }^{5}$ \\ ${ }^{1}$ Department of Medical IT and Marketing, Eulji University, Seongnam, Korea \\ ${ }^{2}$ Department of Information and Comminication, Dong Seoul University, \\ Seongnam, Korea \\ ${ }^{3}$ Department of Broadcasting Production, Kookje University, Pyeongtaek, Korea \\ ${ }^{4}$ Department of Electronic Engineering, Gachon University, Seongnam, Konea \\ ${ }^{5}$ Department of Internet, Chungwoon University, Incheon, Kored \\ ${ }^{1}$ (keylee@eulji.ac.kr,tpffldkfma@naver.com,lk04@eulji.ac.kr), ${ }^{j j j k a n g @ d u . a c . k r, ~}$ \\ 3ysim@kookje.ac.kr, ${ }^{4}$ csj0717@gachon.ac.kr, ${ }^{5}$ jjpark@chungkoon.ac.kr \\ *Corresponding Author: lk04@eulji.ac.kr
}

\begin{abstract}
People listen to music according to their emotions. For example, people listen to sad music when melancholy and fun music when amused. But it is hard to search music similar to their emotions. The mobile phone has information which is created by people who use it. It means the mobile phone has his or her emotions. So, to use a mobile phone, we analyze the users' emotions and recommend it to them? Music applies Valance-Arousal Model for classifying emotions and utilized Korean morpheme analyzer to understand its meaning by extracting verbs. This system is listening to music followed by the mobile phone's text message. In this paper, we designed and implemented thentelligent music recommender system.
\end{abstract}

Keywords: Moblle Phone, Korean Morpheme Analyzer, Valance-Arousal Model, Emotions-Music

\section{Introduction}

According to a surye of mobile phone usage rate in the second half of 2012, people use mobile phones for 3.4 hours a day and sending text messages occupies $20.3 \%$ of their entire using time[1]. From this, we can understand that mobile phones are frequently used in daily life[2-5].

Also, musie influence people's feeling to musical element by prompting people's inner word [6]. This indicates that users listen to music for their feelings.

But in daily life there is an inconvenience that people has to evaluate music one by one when they listen to the music which fits their feelings. Thus, this paper suggests the system that recommends music for feeling in the mobile phone. To understand the user's feeling, the contents of the messages and the saved lyrics in the server are used in Korean morpheme analyzer of Lucene Library to extract verbs and are emotionally classified. 


\section{Related Research}

\subsection{Valance-Arousal Model}

As in Figure 1, we utilize Valance-Arousal(V-A) Space for extracting user's emotional information using mobile phone. V-A model make up for 2 dimensions the Valance axis and Arousal axis. (+) Axis represents the pleasant group and (-) axis the unpleasant group in the Valance axis. (+) Axis represents the activation group and (-) axis the deactivation group[7].

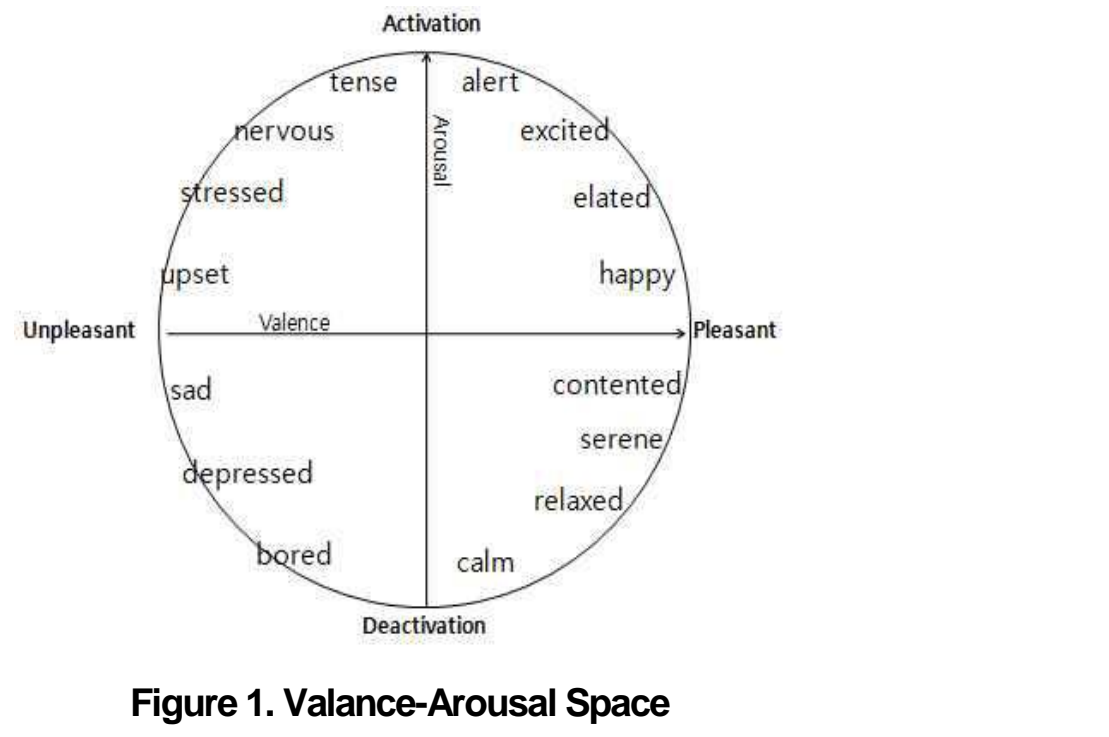

\subsection{Lucene Library}

Lucene was first developed by Doug Cutting and currently is a high performing IR(Information Retrieval) Libary transferred to Jakarta Project. Lucene is not a complete software program but is provided by simple library of Java and is used for importing JAR files.

Lucene is a type of searching tool box and we can make searching programs using it. We cannot apply binary files directly to Lucene but can apply it after converting it to a form of string. Therefore, it is possible to handle documents such as Word files, HTML files, and etc. which is capable of extracting general text files [8].

\subsection{Korean Morpheme Analyzer}

Korean Morpheme Analyzer must consider irregular predicate, word generation rule, compound word, spacing, part of speech system, grammatical morpheme, syllable system, and etc.

Korean Morpheme Analyzer is largely divided into followings. Morphological analysis model of types that are unsupervised probabilistic model and subordinate model. Analysis algorithm based on rule, dictionary and corpus. Morphological direction of bottom-up \& parallel, top-down \& predictive. Left-to-right analysis, word search direction of right-to-left analysis, 2 way analysis, concatenate restriction \& formation of word. Morphological concatenate restriction using connection information $\&$ connection information table. Inspection including unit morpheme or concatenate 
morphemes, units of morphological process that are syllable and phoneme, units of input documents that are word, sentence and paragraph.

Currently, a perspective of Korean Morpheme analyzer takes account of methods using contextual information word by word in sentence[9].

\subsection{Building of Sentiment Lexicon}

By referencing 'Making a List of Korean Emotions Terms and Exploring Dimensions Underlying Theme' by In-Go Park and Kyung-Hwan Min[10], build sentiment lexicon. We build the sentiment lexicon sorted by researcher the originality that is how proper sentiment word is, pleasant-unpleasant feeling that is the more score the more pleasant degree, vitalizations that is the more score the more vital degree. In this paper, we supposed if pleasant-unpleasant feeling is high, pleasant is high in V-A model and if vitalization is high, activation is high.

\subsection{Logcat}

Logcat is a wide use of package to know $\log$ of Implementing application and information of error in eclipse[11].

\section{System Design}

3.1. System Flow Chart
Figure 2 shows system flow chart ging paper.

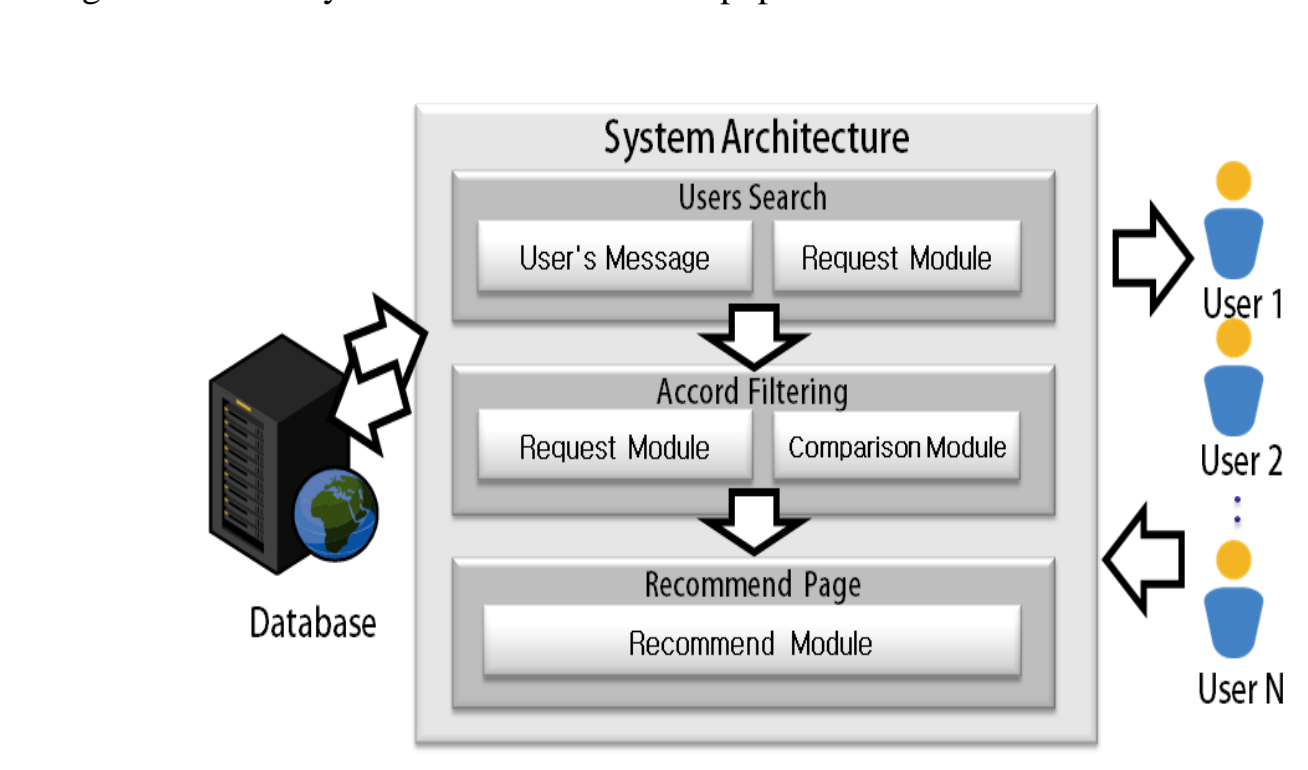

Figure 2. System Flow Chart

When implementing mobile application, the message is transmitted to the server by using the Korean morpheme analyzer. The server extracts only verbs in message received by the user. Then, the data is searched through the database and extracts sentiment data using V-A model. Music is recommended to the users which sentiment data is equal with sentiment data of extracted verb. 


\subsection{System Architecture}

Figure 3 shows System Architecture in this paper.

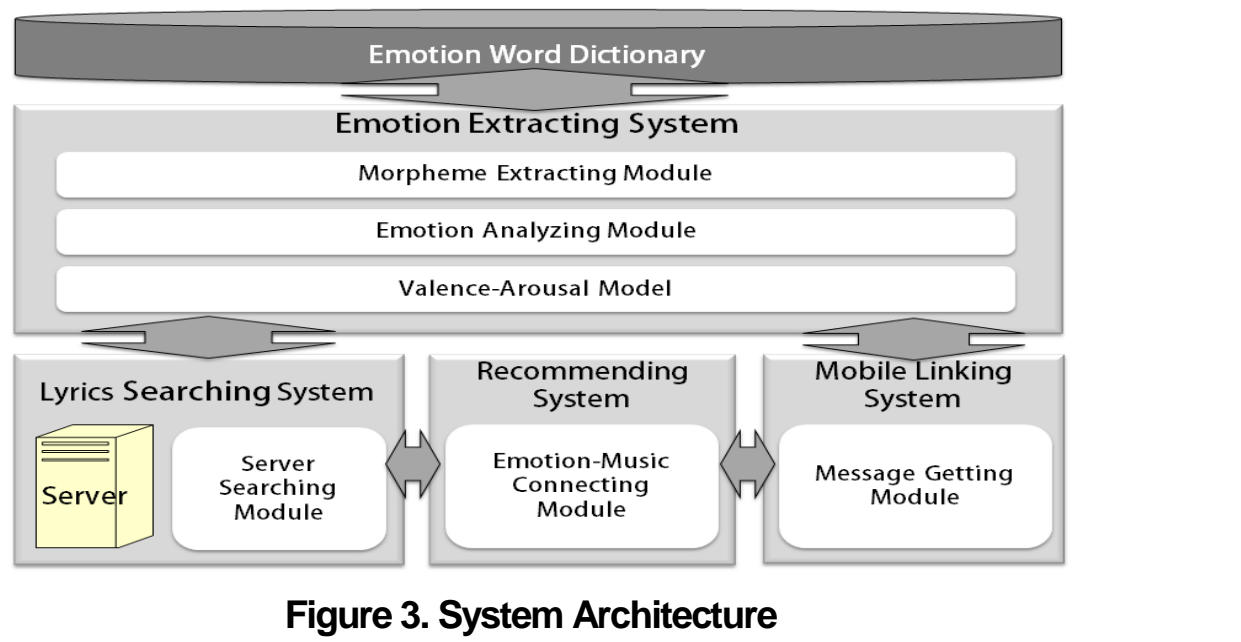

Build sentiment word lexicon by referencing Making a List of Korean Emotions Terms and Exploring Dimensions Underlying Theme'[6]. If new lyrics entered to the server, it is saved in Server Searching Module of Lyries Searching System. And if the user implements mobile applications, the contents of the text message is obtained through the Message Getting Module of Mobile Lirking System. Data that is received by Lyrics Searching System and then Mobile Linking System is refined and classified by the Emotions Extracting System. Obtained data from the Emotions Extracting System's Morpheme Extracting Module exteress verbs and makes it into a type of 'be $\sim$.

Analyze verbs with the Emotions Word Dictionary in Emotions Analyzing Module. Look for verb's average of pleasant-unpleasant score and vitalization score in database of server. Based on it, after classifying context of text messages and music through emotions in Valence-Arousal Module, music is classified with sentiment category and saved in database. Afterwards, the user is recommended music as same as sentiment category of text messages in Emotions-Music Connecting Module of Recommending System.

\section{System Implementation}

Table 1 shows system in test. The hardware specification is Intel ${ }^{\circledR}$ i5 $2.50 \mathrm{GHz}, 8 \mathrm{~GB}$ RAM and use Windows 7 SP1. Also, Lucene which is open source JAVA searching engine wasused for the test. Used mobile phone is Samsung Galaxy Note2.

Table 1. Experiment Environment

\begin{tabular}{c|c}
\hline RAM & $8 \mathrm{~GB}$ \\
\hline \multirow{2}{*}{ Programming Language } & Java \\
\cline { 2 - 2 } & Android \\
\hline Database & MySQL \\
\hline Search Engine & Lucene \\
\hline CPU & Intel i5 $2.50 \mathrm{GHz}$ \\
\hline
\end{tabular}


Used words are 76 typical words in 'Making a List of Korean Emotions Terms and Exploring Dimensions Underlying Theme'[6]. In database of server word is saved with each vitalization score and pleasant-unpleasant score of word. Vitalization score and pleasant-unpleasant score in V-A model have positive integer, so convert it to space by minus average of each degrees. Figure 4 shows algorithm of applying V-A model.

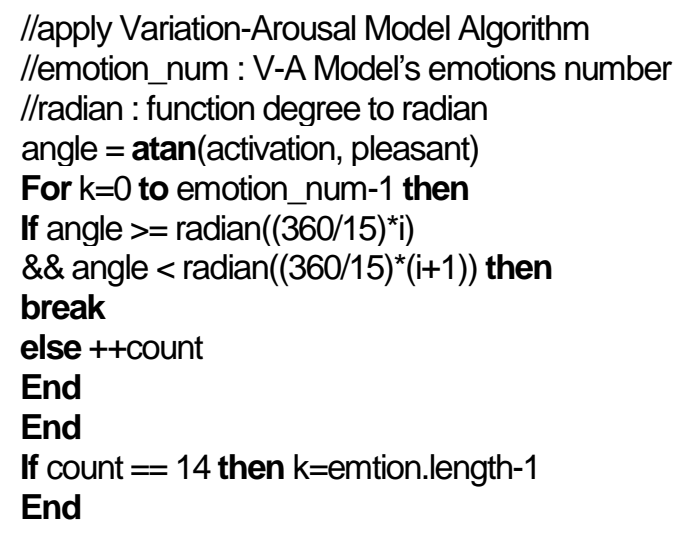

Figure 4. Applying V-Amodel Algorithm

Verbs are extracted from user's text messages. After, find the score of pleasantunpleasant and vitalization of the verb, and if extracted verbs are numeral figure the average of those. Look for degree of radian in application of atan function in average of vitalization divide pleasant-unpleasant. Thensave arrays in order of 'Happyness'. In VA model, if the degree of radian decreases, it is close to 'Content'. Choose the limitations of using those

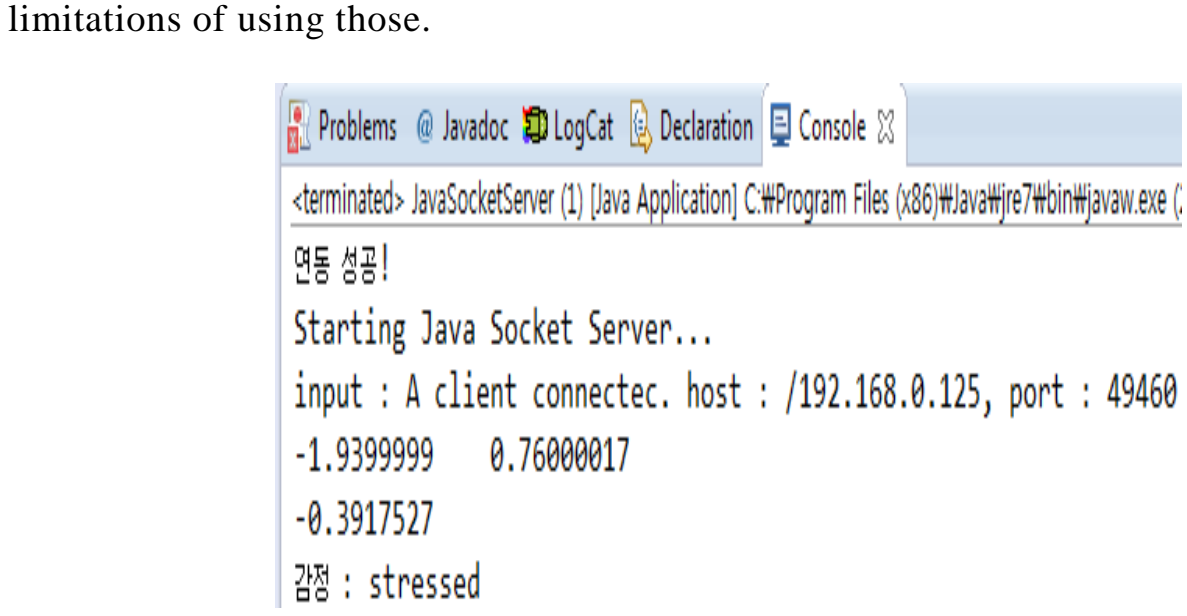

Figure 5. Console of Result in Server

Figure 5 is a result of algorithm in server using a text message of 'deep sorrow'. ' 0.9399999 ' is a score of pleasant-unpleasant, ' 0.76000017 ' is score of vitalization and ' -0.3917527 ' is radian. Then print out 'Stressed' of emotions. 
Table 2. Postclassified Music

\begin{tabular}{cc}
\hline Music & Emotions \\
\hline Noon wi qot & sad \\
Miryunhae ahjicdo saranghae & \\
Manyakae & happy \\
FANTASTIC BABY & \\
Magiccarpetride & \\
Yozm noe malya & calm \\
Noe ibuyl na ahjick &
\end{tabular}

Table 2 shows music that is classified by lyrics. This is music of 3 emotions that is happy, sad, and calm of entire 15 emotions in V-A model. Music is saved with emotions category in database of the server.

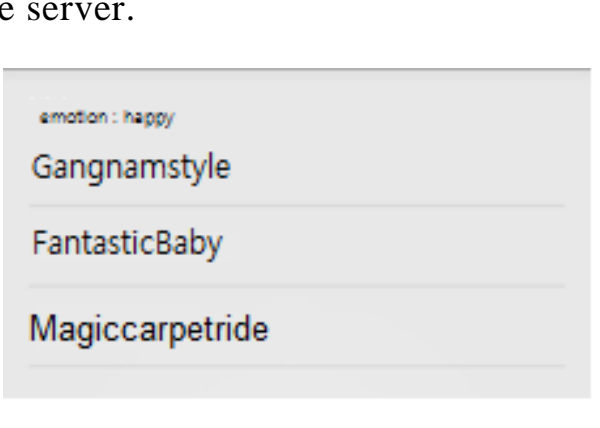

Figure 6. Implemented Result of Application for Emotions 'Happyness'

Figure 6 shows implemented result of sentence 'I'm happy'. It is classified well and user is recommended music of 'happy' category.

\section{Performance Evaluation}
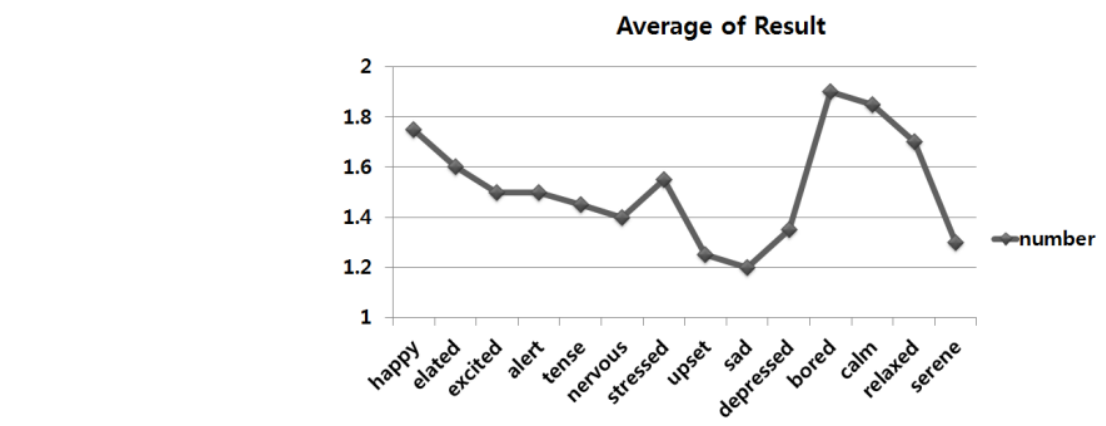

Figure 7. Result of Experiment 
The experiment was processed by 20 people with 30 text messages in three days. Figure 7 shows count of average of text messages which were recommended by each emotions. Average of text messages that were recommended to music was 22.5 cases. Most frequent emotions is 'bored', and next was 'calm' and 'relaxed'.

User's satisfaction score was 6.5. It shows users are mostly satisfied with this system.

\section{Conclusion}

People listen to music according to their emotions. But it is inconvenient to search music like this. So we implement an application extracting emotions using user's text messages in their mobile phone. User's satisfaction score is 6.5 which quite high. However, information is minimum to classify emotions with lyrics. In the future, considering lyrics as well as many other contents, we will postclassify music. Arso, we will consider other media to recognize user's emotions.

\section{Acknowledgements}

This article is a revised and expanded version of a paper entitled [A study on the Smart Music Recommendation System] presented at International Symposium on Advanced and Applied Convergence held on November 14-16, 2013 at Seoul Korea.

\section{References}

[1] Korean Internet \& Security Agency, Survey of Mobile Phône Usage, (2012).

[2] K. Y. Lee, J. J. Kang, A. Kim, M. J. Lim, Y S. Im, S. J. Choi and J. J. Park, "Design and Implementation of the Intelligent Music Recommender System on the-Mobile Phone", Advanced and Applied Convergence Letters(AACL), vol. 1, (2013), pp. 142-143.

[3] W. R. Jo, S. M. Kim and B. G. Joo, A Study on Firmware Optimization Approach of Smart Phone", Journal of the Institute of Internet, Broadcasting and Communication (JIIBC), vol. 12, no. 5, (2012), pp. 177-183.

[4] D. Nguyen and S. Y. Oh "An Architecture fo-Efficient RDF Data Management Using Structure Index with Relation-Based Data Partitioning Approach", International Journal of Internet, Broadcasting and Communication (IJIBC, vol. 5, no. 1, (2013), pp. 14-17.

[5] H. N. Jang and J. P. Jeong, "Analytical Approach of Multicasting-supported Inter-Domain Mobility Management in Sensor-based Fast Proxy Mobile IPv6 Networks", International Journal of Advanced Smart Convergence (IJASC), vol. 1, no. 2, (2012), pp. 1-11.

[6] A. R. Choi, "The Effect of Listening to Music with Lyrics on The Mood State of Workers", Master's Thesis, Sookmyung Women's Oniversity, (2012).

[7] S. Christian, E. Martin, S N. Silke, S. Gig and H. Andreas, "Emotions Detection: Application of the Valence Arousal Space for Rapid Biological Usability Testing to Enhance Universal Access", The Journal of Institute of Human-Computer Interaction(JIHCI), vol. 5, no. 1, (2009), pp. 615-624.

[8] D. Yang, "Implementation of Blog Search Engine using Lucene Library, Master's Thesis. Daejin University, (2009)

[9] S. S. Kang "Korean Morphological Characteristics and Morphological Analysis," The Journal of Computing So ence and Engineering(JCSE), vol. 13, no. 8, (1994), pp. 47-59.

[10] J. Park and K. H. Min, "Making a List of Korean Emotions Terms and Exploring Dimensions Underlying Them", Korean Journal of Psychological Association(KJPA), vol. 19, no. 1, (2005), pp. 109-129.

[11] D. H. Kim and S. W. Park, "Logical Sensor Framework for Recommendation in Mobile Devices", Korean Computer Congress(KCC), vol. 39, no. 1, (2012), pp. 149-151. 


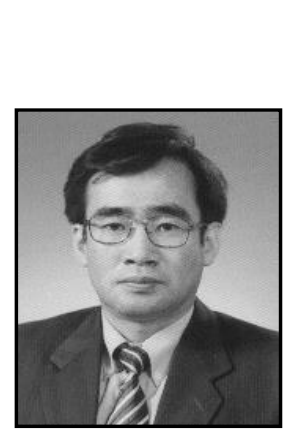

\section{Authors}

Ki-Young Lee received his B.S. degree in Computer Science at Soongsil University in 1984. In 1988 and 2005, he received M.S. and Ph.D. degrees in Databases at Konkuk University, respectively. From 1984 until 1991, he worked for Korea Institute of Ocean Science \& Technology(KIOST) as a researcher in Data Information \& Processing department. He is currently a professor at the department of Medical IT and Marketing at Eulji University. He is also the head of department of S/W development in Bio-Meditech Regional Innovation Center at Eulji University. $\mathrm{He}$ is the director of the Korea Institute of Internet, Broadcasting \& Communication(IIBC), and the director of the Korea Electronics Engineers(IEEK). His research interests inclitide spatial databases, geographic information systems (GIS), location-based services (LBS), u-Healthcare, ubiquitous sensor network (USN), moying objects databases, and telematics etc.

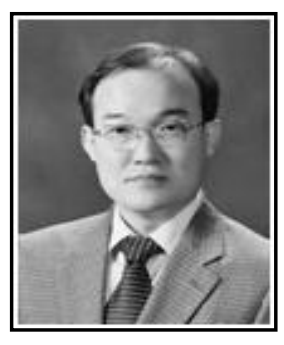

Jeong-Jin Kang is currently the faculty of the Department of Information and Communication at Dong Seoul University in SeongNam, Korea since 1991, and currently the President of the Korea Institute of Internet, Broadcasting \& Communication(IIBC). During 3 years from Feb. 2007 to Feb. 2010 he worked as a Visiting Professor at the Department of Electrical and Computer Engineering, The Michigan State University He was a lecturer of the Department of Electronic Engineering at (Under)Graduate School(1991-2005), The Konkuk University. DN. Kang is a member of the IEEE Antennas and Propagation Society (AEEE AP-S), the IEEE Microwave Theory and Techniques Society(IEEE MTT-S), and a member of the Korea Institute of Internet, Broadcasting \& Communication(IIBC), Korea. His research interests involve Smat Mobile Electronics, RF Mobile Communication, Smart Convergence of Science and Technology, RFID/USN, u-Healthcare and ultrafast microwave photonics, as well as GIS, LBS, moving objects databases, and telematics etc.

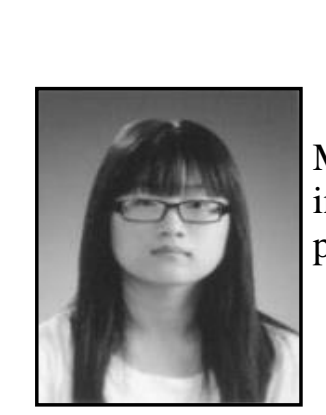

Aleum Kim is currently a student in the department of Medical IT and Marketing at Eulji University. Her research interests include artificial intelligence(AI), search engine, algorithm, android programming, mobile programming, etc. 


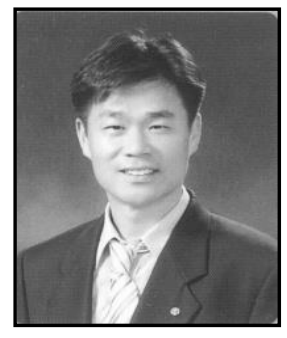

Myung-Jae Lim received his B.S. degree in Computer Science in 1989. In 1991 and 1998, he received M.S. and Ph.D. degrees in Software Engineering at Chung-Ang University, respectively. From 2009 until 2010, he served as the judging panel for Korea Information Processing Society (KIPS) paper. He is currently a professor at the department of Medical IT and Marketing at Eulji University. He is the director of the Korea Institute of Internet, Broadcasting \& Communication(IIBC). Since 2009, he has been serving as the advisor for performance review at SungNam City, South Korea. His research interests include Information Retrieval, USN, u-Healthcare, and Social Commerce etc.

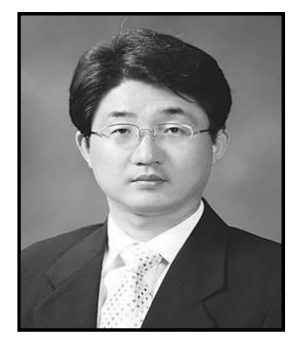

Yong-Soon Im received his B.S. degree in Electronic Engineering at Sungkyunkwan University in 1988. In 1993 and 1999, hereceived M.S. and Ph.D. degrees at Sungkyunkwan University, respectively. From 1988 until 1990, he worked for LG Electronics âs a researcher in Video Camcorder. He is currently a professor at the department of Broadcasting Production at Kookje University.He is the Vice President of the Korea Institute of Internet, Broadcasting \& Communication(IIBC). His research interests include Image processing, Image communication and mobile contents etc.

Sung-Jai Choi was born in cheonan si, chung cheong nam - Do,

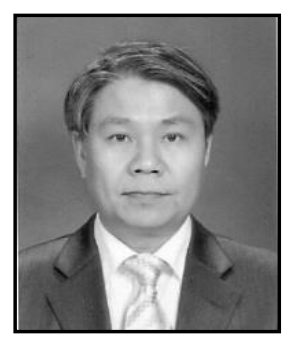
Republic of Korea. He received the B.S. degree in electronics from Chungnam National University, Daejeon, Korea, and M.S. degree in electronics from Hanyang University, Seoul, Korea, and Ph.D. degrees in electronic engineering from MyongJi University, Yongin, Gyong gi Do 1 n 1981 and 1985and 2004 , respectively. Since 1988, he has been a professor at the department of Electronic Engineering of Gachon University w the's main interests involve semiconductor manufacturing technology electronic circuits analysis and fault testing, superconductor , Smart Mobile Electronics, RF Mobile Communication , Smart Convergence of Science and Technology, RFID/USN AND UHeqlithcare, as well as New Media Service. Professor Choi is a Member or the Korea Institute of Internet, Broadcasting \& Communication(IIBC), Korea, The Korean Information Processing Society (KIPS), The Institute of Electronics Engineers of Korea (IEEK) and Korean Institute of Electrical and Electronic Material Engineering (KIEEME)

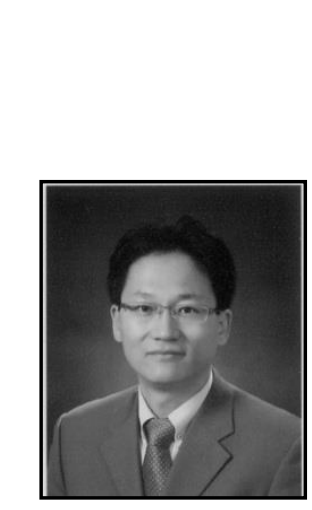

Jong-Jin Park received the B.S., M.S., and Ph.D. degrees in electrical engineering from the Yonsei University, Seoul Korea, in 1989, 1991, and 1997, respectively. He is currently associate professor at the department of Internet of Chungwoon university in Incheon from 1997. His current research interests include Artificial Intelligence, Fuzzy modeling, Mobile Computing, andEmbedded system etc. 
International Journal of Smart Home

Vol.8, No.2 (2014)

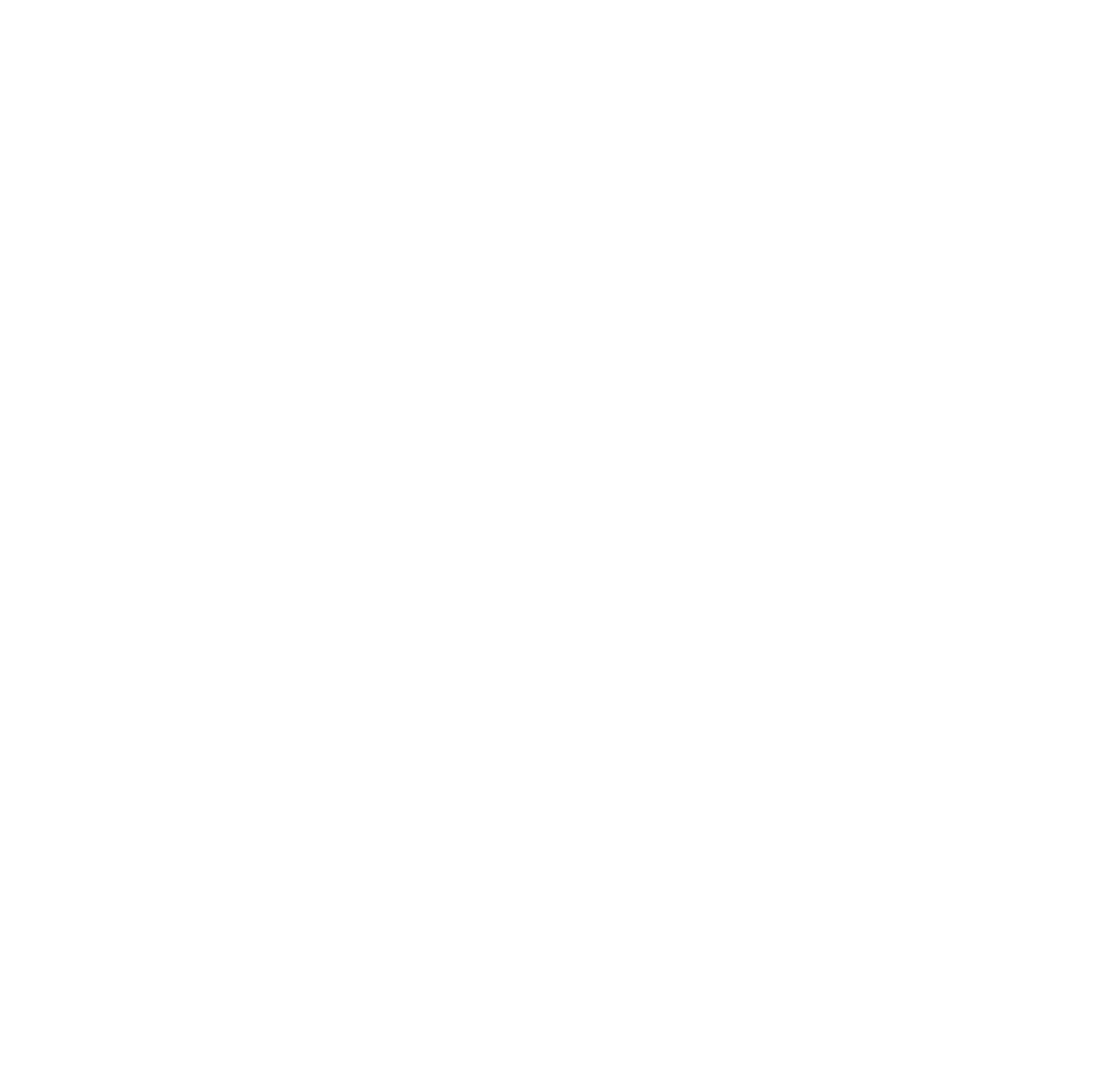

